\title{
STUDENTS AS MULTIMEDIA COMPOSERS
}

\author{
Kenneth E. Hay,' Mark Guzdial. ' Shari Jackson, ${ }^{3}$ Robert A. Boyle ${ }^{3}$ and \\ Elliot Soloway \\ 'Indiana University School of Education. Indiana University-Purdue University at Indianapolis. \\ 902 West New York St, Indianapolis, IN 46202-5155, U.S.A.. 'School of Engineering and Computer \\ Science. Georgia Technological University. Georgia. U.S.A. and 'Highly Interactive Computing \\ Environments Research Group, The University of Michigan, Ann Arbor, MI 48109, U.S.A. \\ [e-mail: khay@indyvax.iupui.edu]
}

(Received I June 1993: accepted 5 April 1994)

\begin{abstract}
This study is distinguished by distancing itself from the type of multimedia research that looks at the effects of multimedia instructional units on students. The approach. instead, is within a constructionist learning paradigm and the research task is to take the first steps to begin to know what we don $t$ know about the student creation of multimedia documents.

The outcome of preliminary research on the use of MediaText, a multimedia composition tool. with high school students across several disciplines indicates from an analysis of document level and link level data on 62 student-produced multimedia documents across individual students and assignments that students unfamiliar with multimedia tend to apply their existing writing skills to produce annotated text rather than integrated compositions, in which the links are clearly part of the structure of the data.
\end{abstract}

\section{RESEARCH AND THEORY OF MULTIMEDIA IN EDUCATION}

The notion of applying multimedia to education has been around for a number of years. There are a large number of educational multimedia products on a variety of topics produced, sometimes by teachers, but most of the time by professional or academic instructional designers. The educational power of this new medium is indeed extremely thought provoking. However, students have often been fixed at the stationary endpoint of these instructional units, the targets of the instructional designers' objectives. Thus, the student's position in this multimedia system has changed very little from the old CAI days. This paper discusses the use of multimedia to empower students with the ability to express their thoughts in an ever expanding range of "media avenues". Specifically, we look at the documents students created using the software. MediaText, which empowers students with the expressive capabilities of multimedia, and little of the previously associated up front cost of learning a programming language or professional level, multimedia packages to manipulate the different media.

There is a long history of research on the effects of media on learning, much of it dealing with a single medium (for reviews of the literature, see $[1-4]$ ). This research has focused on the effectiveness of various media in learning and instruction. Researchers have extended this single medium research to the frontier of multimedia research. They explore what functionalities of multimedia systems are beneficial to learning from instructional units they produce. We have broken from this line of research by using two distinctions that have been recently introduced into the educational research literature.

The first distinction we use is made by Papert [5] between instructionism and constructionism. This distinction forms one of the cornerstones of our notion of how multimedia should be used in education. Instructionism is the notion that students are passive receptacles for the knowledge that the teacher or some other instructional medium imparts to them. Papert takes two steps away from this notion, first with constructivism, and second with constructionism, where:

"The word with the $v$ expresses the theory that knowledge is built by the learner, not supplied by the teacher. The word with the $n$ expresses the further idea that this happens especially felicitously when the learner is engaged in the construction of something external or at least sharable ... a sand castle. a machine, a computer program, a book." $[5$, p. 3] 
The second distinction we use is made by Salomon et al. [6] between the effects on student cognition with technology as opposed to effects of technolog.: In studying the effects of technolog.' researchers gather evaluation data after the student stops working with the technology. They explore the impact or residue that is left behind by the technology. When studying the effects with technology, researchers evaluate the student/technology system to understand how the empowered student changes.

Currently, the majority of educational research on multimedia explores the effects of technology within an instructionist paradigm. In this paradigm, students explore multimedia webs of information or take multimedia tours. However, we feel that the most fruitful use of multimedia in education lies in looking at the effect on the student empowered with techology within a constructionist paradigm. In this paradigm, students are empowered to actively construct multimedia artifacts. They are empowered with sophisticated software tools that allow them to construct multimedia artifacts as readily as they would build a "sand castle". Our strategy in reviewing the literature on multimedia in education will be to comb the previous media research that is within the instructionism paradigm. This will help inform the use of technology that empowers students within a constructionist paradigm. Then we will turn to the pioneers in the study of students as multimedia composers.

\section{Multimedia research}

There is evidence in previous research that lends support to several benefits of learning with media-cognitive, metacognitive, and motivational. Recent research on media learning investigates how media can assist students in cognitive and metacognitive tasks. Work by Neuwirth and Kaufer [7] and by Greeno [8] suggests that media caln assist students in encoding information through the richness of the symbol systems used by various media. This is done by presenting concrete representations of abstract concepts, thereby assisting students in incorporating these new concepts into their knowledge bases and developing more complex mental models. In addition, Salomon has proposed a Media Attributes Theory [2] which tries to explain the likelihood that media representation will assist a student's learning by its "distance" or difference from the student"s own representations. The "closer" the two, the easier it is for a student to process the media information. While still somewhat within the instructionist paradigm, we feel this research can be used to better support having the students find or create representations on their own and assemble them in a manner meaningful to them. We contratst this to what would occur if the students simply watched a multimedia presentation constructed by their instructor.

The metacognitive benefits of media have been described in terms of the transfer of knowledge learned in one situation to a different situation. Studies of media seem to indicate that transfer may occur only under limited, specific conditions [2,3]. Kozmal [4] argues that, due to the attributes of recursion and interactivity, multimedia systems may more powerfully promote this metacognitive skill over other media in that such systems can focus student thinking on the interconnections between ideas. However, in these studies, the student is the passive consumer of an exploratory multimedia system which is once again within an instructionist paradigm. (There are writers who argue that by the movement through the exploratory multimedia system the consumer is active instead of passive. It is our contention that this is not much different from the "activity" of choosing which television channel to watch, or which book to read, and is fundamentally different from the activity of composition.)

The transfer issue within our constructionist paradigm will be a matter of continuing research and is not the focus of this research. However, constructive multimedia systems, with the type of foundational research that this study presents, may provide powerful avenues for transfer for the following reasons:

- constructive multimedia systems, by allowing for the simultaneous construction of multiple media formats, free students to express themselves and think with different media about the same idea;

- constructive multimedia systems are able to empower students to author and think with media and their interconnections. This puts students into an authoring environment unbeknownst to them in the past; 
- constructive multimedia systems that allow easy composition of multimedia documents are able to provide a vehicle to bridge from the abstract to the concrete or from one context to another.

In terms of motivation, both Bransford et al. [9] and Scardamalia et al. [10] mention the positive effect students display towards learning in media/computer environments. The research by Clark and Salomon [2], mentioned above, suggests that this may be more distracting than beneficial. However. Clark and Salomon note a change in media research (one reflected in the work of both Bransford and Scardamalia) in which the media and student interact to construct meaning. rather than the student passively receiving information. Thus the positive effect demonstrated in more recent studies may have a much different cause than those of the past. The "active learning" aspect of many computer/media systems may also foster a sense of personal control, as well as evoking a sense of challenge and curiosity in the student [11].

MediaText requires the student to be active in the same way a word processor or a paper and pencil do in writing a paper. Furthermore. it provides students with choices which were previously unavailable. They have the ability to "say" what they want in movies, animations, pictures or sounds, as well as with text. Having established this research within an instructionist paradigm and bridging it to a constructionist paradigm. we will now turn to similar efforts.

\section{Rescarch in student composition of multimediat}

The view of student as multimedia constructor instead of consumer is becoming a popular one. One only has to look within educational technology journals that are aimed at teachers, such as the Computing Teacher, to find many ativities where students are construction multimedia documents (i.e. $[12,13])$. The catalyst that started the movement to empower non-programmers, including students, to compose multimedia was the Apple Corporation [14] development of HyperCard, the first readily accessible multimedia composition tool. Most of the research on student composition of multimedia has been conducted when students were utilizing HyperCard [15-18]. There have been other past and ongoing projects that are developing multimedia composition tools: Multimedia Works by Pea [19]. StoryShow by Reilly [20], and the Production Console in The Civil War Interactive Project [21]. Most of these studies struggled with the isste that Turner and Dipinto [16] articulate:

in order for students to become hypermedia authors, they must learn not only the content they will present in the hypermedia document but also the tool skills they need to use the hypermedia software.

In one study, Reed and Rosenbluth [18] taught and studied a Hyper-Humanities course where students had 17 2-h sessions on HyperCard and 13 2-h sessions on researching the humanities. Despite this struggle and the tendency of projects to be based in after school clubs or summer programs, researchers report overcoming these problems with encouraging results. Pea [19] states that students did "find the process engaging and motivating" and engaged in content-rich activities with their multimedia composition tools. This has been confirmed with studies involving student from the second grade [20] to Occupational Therapy students in the university. Farrow [17] found that " $74 \%$, [of the students] indicated that the learning experience was valuable". The "linking nature of HyperCard" fostered an "increased awareness of the interrelatedness of these factors" affecting values of reported decades [18]. The multimedia environment promoted "creating rich conversational artifacts for discussion and presentation" [19] and the possibility of rich collaborative work [20].

Nicol [15] makes the observation that students "need new and more engaging hypermedia models" and that without them they flounder. The burden of the technology was evident in Reilly [20], where difficulty with imaging technology caused the student to do litte or no editing of images once they were in the computer: "it was usually saved as-is and rarely rejected or retaken". This differed from the sound which was easier to produce and thus was "more often rejected and re-recorded".

In summary, there is evidence in the literature on media learning to suggest that actively involving 
students in constructing knowledge from multimedia systems can lead to cognitive, metacognitive, and motivational gains. We have found Nicol [15] leading the way into the realm of analyzing multimedia documents created by students in a constructionist learning environment.

We must start with some basic questions that need to be addressed before the above issues can be examined in detail. These questions are:

- Do students, using this program, write in a different manner or style from those using just a word processor?

- Do they make use of the various media tools available to them to incorporate concepts for non-text media into their documents?

- What does multimedia composition look like?

- What are some characteristics of multimedia compositions?

Because we, as researchers, are just beginning to understand student multimedia compositions, we felt it necessary to give students the freedom to do what they were capable and interested in doing, rather than prescribing the manner in which they were to write. In the rest of this paper, we describe both the tool students used (MediaText) and the nature of the students' work in multimedia writing. Once we understand what students are capable of doing with MediaText, we will be better able to investigate the possibilities that multimedia composition has for learning.

\section{MEDIATEXT: A MULTIMEDIA COMPOSITION TOOL}

The Highly Interactive Computing Environments (HiCE) group at the University of Michigan has developed MediaText, a software multimedia composition tool. To use an analogy, MediaText is to various media as a word processor is to text. It allows the user to easily create documents containing text, graphics, animations, sounds, and links to devices such as videodisk players. This multimedia composition tool was designed to give the student access to multimedia without the overhead associated with other programming languages. The design also includes a readily accessible metaphor, the word processor. MediaText was developed with the intent of creating a simple tool for Macintosh computer users to compose multimedia documents. The main features of the program are:

- A standard word processor which comprises the left three-quarters of the document window and allows for multiple styles, sizes and fonts.

- A Media Margin on the right one-quarter of the document window next to the word processor which contains MediaLinks. The Media Margin maintains the spatial relationship between the MediaLinks and text in the word processor whenever the text is scrolled up or down or additional text is added to the document, MediaLinks remain next to the text where they have been placed.

- The MediaLinks are represented by icons which connect another medium to the current document. Clicking these icons activates the MediaLink that sends the reader to another medium. The MediaLinks are fully editable, and may be dragged within the Media Margin, as well as cut, copied, or pasted within a single document or among several documents as though the MediaLinks were text in a word processor.

- The MediaLinks Menu is an additional pull down menu on the menu bar through which "Media Workshops" are accessed. The Media Workshops are used to create the different MediaLinks. These Workshops contain tools for creating, selecting and/or editing other media documents which are then linked to a MediaText document.

A sample screen of a Media Text document is shown in Fig. 1.

The following is a brief explanation of the Media Workshops and their features.

- The Videodisk and Compact DiskWorkshops incorporate controls for a computer-controlled laserdisc (CAV or CLV) or CD-ROM player and allows the author to create Links by selecting the starting and ending frame numbers or time code for a video segment.

- The Graphic Workshop is a built-in drawing-based graphics editor. This Workshop includes the capability to animate drawn objects by recording the path of the object by dragging 


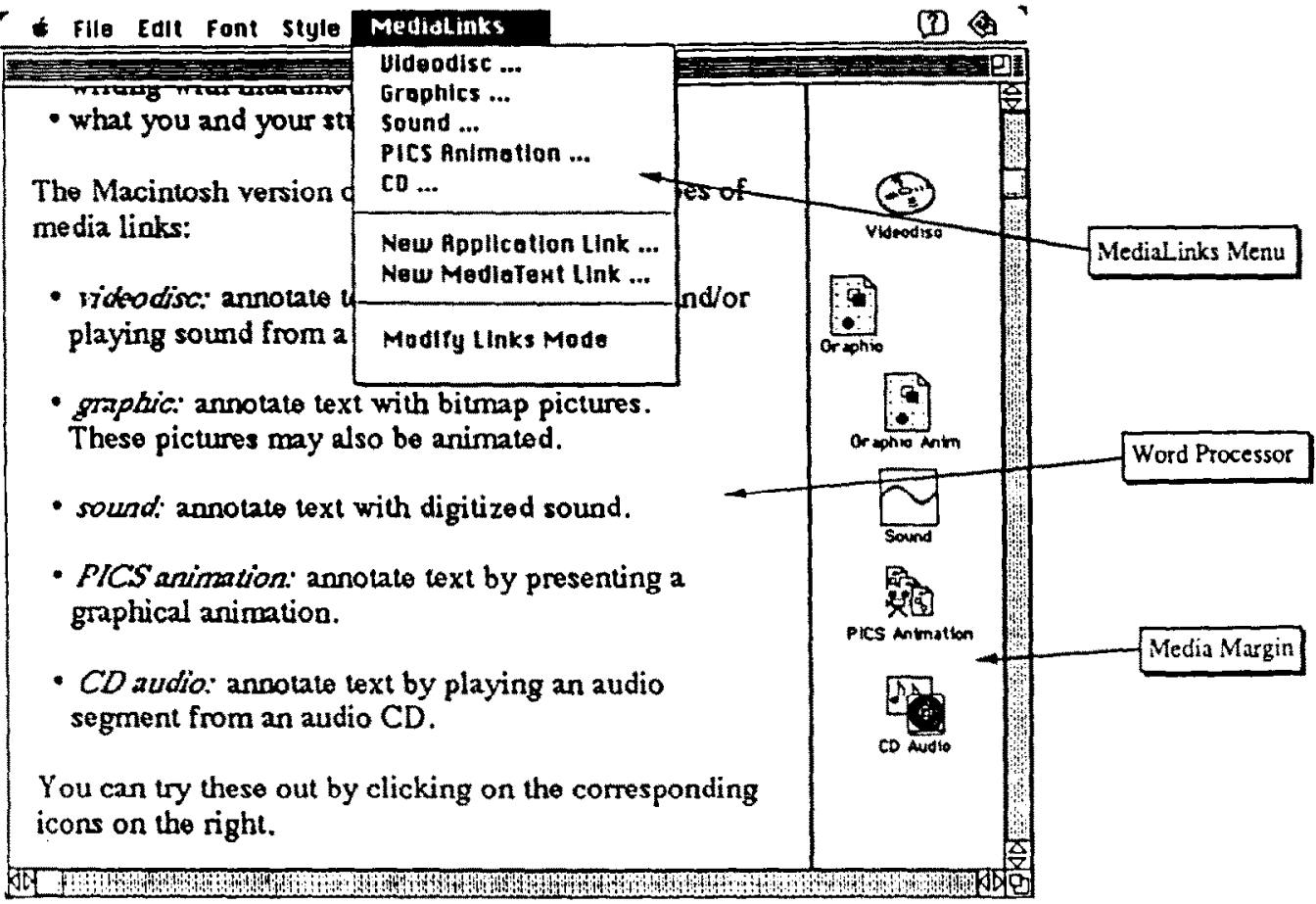

Fig. I. An example of a MediaText screen.

an object around the screen. This can be done with objects or groups of objects simultaneously or individually.

- The Sound Workshop allows the input of digitized sound from a MacRecorder or from a built-in microphone directly into MediaText, as well as, the import of previously created sound liles.

- The PICS Animations Workshop links cell-based animations of the PICS file type to a MediaText document. PICS files can be created by high-end animation software such as Macromedia's Director.

- The Application Link Workshop allows the user to create links to any other document or application.

- The MediaText Link Workshop allows Mediatext documents to be linked to each other. This feature promotes MediaText as a creation tool for hypermedia webs.

\section{DESCRIPTION OF THE STUDY}

MediaText was first used during the 1990-91 school year at Community High School in Ann Arbor, Mich. Community is an alternative public high school in which students elect to enroll. The community which this school serves is a middle to upper-middle class university town, and typically a small proportion of minority students elect to enter this school. Students are admitted to the school on a first-come, first-serve balsis with no other entrance requirements, allowing for a wide range of ability levels and interests. The program at the school focuses on individualized learning programs for students as well as a focus on community and multicultural activities. The computer lab in which the students did their work was equipped with 10 networked Macintosh II and SE computers, all with 4 Mbyte of RAM, 40 Mbyte hard drives, and 19-in. black-and-white monitors. and a LaserWriter. Seven stations were connected to laserdisc players with 13-in. color television monitors. Other equipment available for student use included hand scanners, CD-ROMs. and sound digitization hardware. Support materials included a clip-art library and a library of videodisks ranging from disks designed for classroom use to popular movies. Students brought in 
their own audio compact disks. Students in this high school are required to take a certain number of Community Resource credits as a requirement for graduation. These courses range from community service to activities that explore hobbies or special interests. The computer lab was available to all students, every afternoon from 2 to 5 p.m.

We studied the work of students who used MediaText to meet their assignments in four classes conducted over the course of the school year: English Composition and Physics during the fall semester; Science and Society and Multimedia: Art and Technology during the winter semester.

\section{English Composition}

In the English Composition class, MediaText was used for two assignments by small groups of students working in parallel with the remainder of the class. Students were selected to work with MediaText on a volunteer basis. The student volunteers worked in a computer lab and staffed by a technical support person next door to the classroom. Both groups worked on the same assignment directed by the same classroom teacher. For the most part, these students were inexperienced users of MediaText and had a small amount of time to work with the program for this assignment.

The first assignment tackled by the students was biography. Each student in the class was expected to choose someone they knew and interview them. Five students volunteered to use Media Text for this assignment. The class devoted 2 weeks to the assignment. The students working with MediaText took from 3 dalys to 2 weeks to complete the assignment. Students chose subjects for the biography assignment ranging from friends, to local television personalities, to musicians. After all the students had completed their projects, everyone shared their work with the rest of the class.

Reactions to the multimedia biographies were so positive that 12 students asked to work with MediaText for the second assignment, writing a "how to" document. The teacher chose 10 students to work with Mediatext for this assignment due to the limited number of compulers available. Topics chosen by the students included How To Be Annoying, How To Skip School, and How To Be A Leaf.

Physics

In the Physics class, computers were already somewhat integrated into the curriculum. The Physies teacher had a few years of experience using spreadshects for working with lab data, as well as simulations and microworlds to demonstrate physical concepts. The teacher also encouratged the students to use word processing software in writing their lab reports. The teacher used MediaText in two distinctive ways.

The first method was to use MediaText on an ongoing basis. This was done by dividing the class in half and sending each half to the computer lab 1 day a week under the supervision of a technical support person. Thus, each student spent about 50 min per week working with MediaText. In the computer lab, students were asked to keep a chronicle or journal of what they had learned in class and illustrate this journal with links.

The second method was to use MediaText at the end of the fall semester for a final evaluation. Physics students were given the option of either taking the standard semester final exam, or creating a MediaText document that would discuss and/or demonstrate all the concepts covered during the semester. Students who chose the multimedia option were given a list of topics to cover and were required to use a variety of media to illustrate their final papers.

\section{Science and Society}

The focus of the Science and Society class was to study how scientific and technological improvements affect societal practices and vice versa. Students had the option of electing the class for science or for social studies credit. For one of the papers assigned in class, the students had to focus on the development of a specific type of transportation. Six student volunteers were selected to work in MediaText on this assignment. To complete this assignment, one student traced the development of airships and blimps. 
Table 1. MediaText document summary by class and assignment

\begin{tabular}{cccc}
\hline Class & Assignment & $\begin{array}{c}\text { No. of } \\
\text { students }\end{array}$ & $\begin{array}{c}\text { No. of } \\
\text { documents }\end{array}$ \\
\hline Composition (total) & 15 & $9 *$ \\
Biography & 5 & 4 \\
How To & 10 & 5 \\
Multimedia: Art and Technology (total) & 9 & $45 \dagger$ \\
& Introductory Assignment & 9 & 12 \\
& My Greatest Fear & 7 & 8 \\
& City & 4 & 4 \\
& Videotape & 5 & 6 \\
& A Game Called Hints & 4 & 4 \\
& Independent Work & 5 & 13 \\
Physics (total) & 33 & 24 \\
& Chronicle & 13 & 14 \\
Final & 10 & 10 \\
Science and Society (total) & 6 & 3 \\
Transportation & 3 & 3 \\
Totals for 4 classes & & \\
& II Assignments & 63 & 83 \\
\hline
\end{tabular}

- Not all students produced MediaText documents.

tStudents occasionally produced more thatn one document for an assignment.

\section{Multimedia: Arl and Te'chmology}

The course, Multimedia: Art and Technology focused on using MediatText. This course was offered after school for academic eredit with three members of our research team as instructors. The class was composed of nine students chosen out of a group of about fifteen who expressed interest. These students were chosen on the basis of their schedules to ensure that they would be able to meet after school for $2 \mathrm{~h}$ every week and that they had free access to the after school computer lab. Students in the elective class were assigned five projects over the course of the semester, plus a written assignment, all of which are described below.

- Introductory Assignment was the first assigned project in the course. It was open-ended and designed to allow students to explore the media types available to them.

- My Greatest Fear was the second project assigned. Students were asked to create a document that would convey their feelings of fear to the reader.

- Education Article was the third assignment. Students were instructed to read an article about education and record their personal responses to the various issues in the article.

- City was the fourth document assigned the students. They were asked to create a document about a city.

- Video Tape was the fifth assignment. In this assignment students were to fill in an outline based on class discussion of a videotape that would promote MediaText to an educational audience. The students were given a MediaText document containing a brief outline of the class discussion and were asked to elaborate it.

- A Game called Hints was the final assignment where students created a MediaText document about some object or experience without showing it or naming it directly.

Several of the students in the course produced documents in addition to the assigned ones. These documents were for other classes or out of personal interests. The extra documents are included in the total number for this class and are labeled as Independent Work. Table 1 gives the number of students in each class, the number of documents produced for each assignment, and the document count for each class and each assignment. 
Tabie 2. Final data set summary by class and assignment

\begin{tabular}{|c|c|c|c|c|c|c|c|}
\hline Class & Assignment & $\begin{array}{c}\text { No. of } \\
\text { students }\end{array}$ & $\begin{array}{c}\text { No. of } \\
\text { documents }\end{array}$ & $\begin{array}{l}\text { Average } \\
\text { length } \\
\text { (words) }\end{array}$ & $\begin{array}{c}\text { Average } \\
\text { total } \\
\text { links }\end{array}$ & $\begin{array}{c}\text { No. of } \\
\text { AT } \\
\text { documents }\end{array}$ & $\begin{array}{c}\text { No. of } \\
\text { IC } \\
\text { documents }\end{array}$ \\
\hline \multicolumn{8}{|c|}{ Composition } \\
\hline & Biography & 5 & 5 & 495 & 6.0 & 5 & - \\
\hline & How To & 2 & 2 & 264 & 3.0 & 2 & - \\
\hline & (total) & 7 & 7 & 429 & 5.1 & 7 & 0 \\
\hline \multicolumn{8}{|c|}{ Multimedia: Art and Technology } \\
\hline & Introductory Assignment & 8 & 11 & 518 & 7.8 & 8 & 3 \\
\hline & My Greatest Fear & 7 & 8 & 293 & 4.0 & 4 & 4 \\
\hline & City & 4 & 4 & 410 & 8.8 & 2 & 2 \\
\hline & Videotape & 5 & 6 & 506 & 8.7 & 1 & 4 \\
\hline & A Game Called Hints & 5 & 5 & 87 & 4.6 & 2 & 1 \\
\hline & Independent Work & 4 & 8 & 231 & 4.5 & 5 & 3 \\
\hline & (total) & 9 & 42 & 345 & 6.3 & 22 & 20 \\
\hline \multicolumn{8}{|c|}{ Physics } \\
\hline & Chronicle & 4 & 5 & 326 & 3.8 & 4 & 1 \\
\hline & Final & 6 & 6 & 1201 & 15.3 & 1 & 5 \\
\hline & (total) & 7 & 11 & 803 & 8.4 & 5 & 6 \\
\hline \multicolumn{8}{|c|}{ Science and Society } \\
\hline & Transportation & 2 & 2 & 388 & 4.5 & 2 & - \\
\hline & (totial) & 2 & 2 & 388 & 4.5 & 2 & 0 \\
\hline \multicolumn{2}{|c|}{ Totals for 4 classes } & $22^{*}$ & 62 & 434 & 6.5 & 36 & 26 \\
\hline & II assignments & & & & & & \\
\hline
\end{tabular}

* Several students were enrolled in more than one class.

\section{METHODOLOGY}

This study utilized an inductive methodology $[22,23]$ to analyze the specific types of studentcreated, multimedia documents. It is narrowly defined to examine the products that are created by the student using the technology. This study and its methodology were aimed entirely at understanding what we do not know about the types of multimedia documents students crealte.

This report provides a structural analysis of the multimedia documents ereated during the introduction of MediaText as a medium for high school student multimedial composition across a high school curriculum. Since MediaText differs structurally from programs such as HyperCard, which uses a database metaphor and differs from word processors by the addition of the Media Margin, we focused our attention on characterizing the relations among the elements of the MediaMargin, the links, with the textual elements of the document.

\section{Docaments: of the data set}

Eighty three documents were collected from the students in the four classes: Physics, Composition, Science \& Socicty and Multimedia: Art \& Technology. 21 documents provided no basis for a structural analysis of multimedia composition. They contained only text-no links. They were eliminated from the data set for the purposes of this study. The data set include three students who enrolled in two courses and thus authored documents for both classes. The data set reduced to a total of 62 documents authored by 22 different individuals. Table 2 shows the breakdown of the final data set by the number of students and the number of documents for each of the courses and each of the assignments.

We began by performing open coding [22] of the data by reading and interacting with the documents. This was done by paying special attention to the relationship between the content of the Link and the content of the text. We also gathered quantitative data on the word count and link count, which is also shown in Table 2.

From this analysis, we observed the emergence of two separate categories of writing styles. These categories were later named annotated text (AT) and integrated composition (IC). The initial classification was based on the notion of webs in the literature on multimedia. There was variation 
Table 3. Techniques of integration

\begin{tabular}{|c|c|}
\hline Implicit integration techniques & Explicit integration techniques \\
\hline $\begin{array}{l}\text { JUXTAPOSITION is the technique of placing the link } \\
\text { near content-related text }\end{array}$ & $\begin{array}{l}\text { MENTION is where the name of a related link in the body } \\
\text { of the text is mentioned, often with directions to activate that } \\
\text { link }\end{array}$ \\
\hline \multirow[t]{2}{*}{$\begin{array}{l}\text { TITLING is giving the link a name which relates to } \\
\text { its content }\end{array}$} & $\begin{array}{l}\text { DIRECTING is where the author directs actions the reader } \\
\text { needs to perform to read the document. e.g. clicking. } \\
\text { switching videodisks }\end{array}$ \\
\hline & $\begin{array}{l}\text { SPECIAL TEXT is where the author uses parenthetical } \\
\text { references, boldfice text. indentation, asterisks or other } \\
\text { characters to refer to the link }\end{array}$ \\
\hline
\end{tabular}

in the richness and density of the webs of information that the students created. After looking at the properties of word count, link count, word/link ratios, and the usage of conventional sentence and paragraph structure. there was only one major property [22] for distinguishing between the two different document types. This property was the extent to which a document's text stood alone, i.e. the degree to which the content of the text produced a coherent message without links. There was some correlation between the conventional structuring of the documents' sentences and paragraphs, where AT was more likely to be conventional, however there were a number of IC documents that were conventionally structured.

Annotated text is characterized by its similarity to text-only documents with added links. In AT documents, the text stands alone as a complete work and the links provide parallel information. Thus, the links only serve to broaden the same message. For integrated compositions, the message is distributed between the text and the other media, and often the text is used to describe and explain the content of a link rather than the link illustrating the text.

The documents were divided between two coders. They re-examined the documents and classified each as AT or IC on the basis of these criteria. After classification, they compared each other's encoding. Of the 62 documents, they agreed on the coding of 30 ats clearly of the AT style and 24 of the IC style. The eight remaining documents were ambiguous but they agreed to elissify two of the documents as IC and six as AT. Table 2 shows a breakdown per class and per assignment of the number of $\mathrm{AT}$ and IC documents analyzed.

The eategory of AT proved to have no dimensional property. Thus, if the media links were removed and the text still stood alone, it was said to be AT. Conversely, the IC category varied from exclusive reliance on one integration technique to the utilization of many. Thus, the more a student used integration techniques, the more integrated it was said to be.

Furthermore, during the dimensionalization of the IC category documents, students used a variety of techniques to relate the links and text within their documents. This coarse documentlevel analysis utilizing the AT/IC distinction overlooks the individual differences within a document at the link level. As a further step in the analysis, a second level category system was used to describe more robustly the relationship between the links and the accompanying text.

A category seheme emerged upon a second pass of open coding. These were on different types of connections between the text and the links; integration techniques. Integration techniques are the structural techniques that authors use in composing their documents to alert the reader to the presence of a link. Further integration techniques tended to be either explicit or implicit. Table 3 lists the types of explicit and implicit integration techniques used by the student authors. The following are examples from student documents of the various integration techniques.

Juxtaposition: This was the most common form of integration. Nevertheless, one student put all of the links at the end of the document, in the same content order as that of the text. Another student created a document where the order of content in the links was completely different than in the text.

Titling: For the most part. students would try to name links appropriately, but there were often link names with no apparent connection to the text of a document. An occasional document would contain links that, by their names, did not relate to any section of text. 
Mention: Less often, students would actually discuss the content of the link and the relevance of the link to the content of the surrounding text.

Directing: Students often included directions to the reader on how to read their document. These directions ranged from telling a reader when it was appropriate to play a link or when to switch videodisks.

Overall, how links were integrated with the text varied widely between individual students. Often, students who wrote several documents would show different levels of integration between documents. For one physics student, her final paper, developed over the course of 2 weeks, showed variation in level of integration within that document. The links became more explicitly mentioned and discussed in the text towards the end of the document.

\section{INTERPRETATION}

With the completion of the coding and a firm description of the documents, the research directives were refined to the following:

Explore the extent that the subject matter of the class or the nature of the assignment influenced student use of media.

Explore the differences in student experience levels and the style of multimedia documents.

To address these issues, the data and their related coding schemes were examined in two formats. The first format was to interpret the data on a whole class assignment level. The second format was to interpret the data on a selected group of students and their documents.

\section{Comparison of documents within a particular class/assignment}

For the Composition class, seven documents by seven diflerent students covering two assignments were examined. All of these documents were classified ats AT-zhe text of these documents looked, for the most part, similar to what a classmate might have produced for the same assignment on a word processor without links. These students were relatively inexperienced users of MediaText and had a short amount of time to work on the assignment. Students were in a course context where the model of composition was text-based. We saw these factors having at major contributing influence on the predominance of AT documents.

For the Science and Society assignment, two documents by two different students were examined. The sentences and paragraphs in these documents were structured in a highly conventional manner, and the links mainly supplemented the messalge of the stand-alone text. Again, we hypothesize that because the students in the Science and Society class were working under the same conditions as the Composition students-little experience and little time to work with the program (Fig. 2)-they produced AT documents.

Two assignments were covered in the Physics class. For the chronicle assignment, five documents produced by four different students were examined. These documents were produced early in the students' experiences with Mediatext, though they had the opportunity to work on their chronicles over the course of the semester. Of these five documents, four were classified as AT. Six students chose to complete their final paper in MediaText. By the time they began work, they had already spent approximately ten class sessions working in the computer lab. which included work on MediaText. In addition, these students worked on their documents while the rest of the students were reviewing for their in-class exam: approximately eight class periods. Of the six final documents examined, five were classified as IC (Figs 3 and 4). These documents also contained the most links and were the lengthiest of all the assignments for any class (Table 2), ranging from 10 to 27 links and 683 to 1763 words. This was mostly due to both the number of concepts the students were required to cover and the amount of explanation students gave in describing the content of their links.

The Multimedia: Art and Technology class was the best class to look at trends of long term usage of MediaText because the class worked with MediaText daily and produced the most documents. Ignoring the independent work, where there were eight AT documents and three IC 
Fig. 2. Example of an annotated text document.

Fllo Ealt Font Stylo Mediolinks

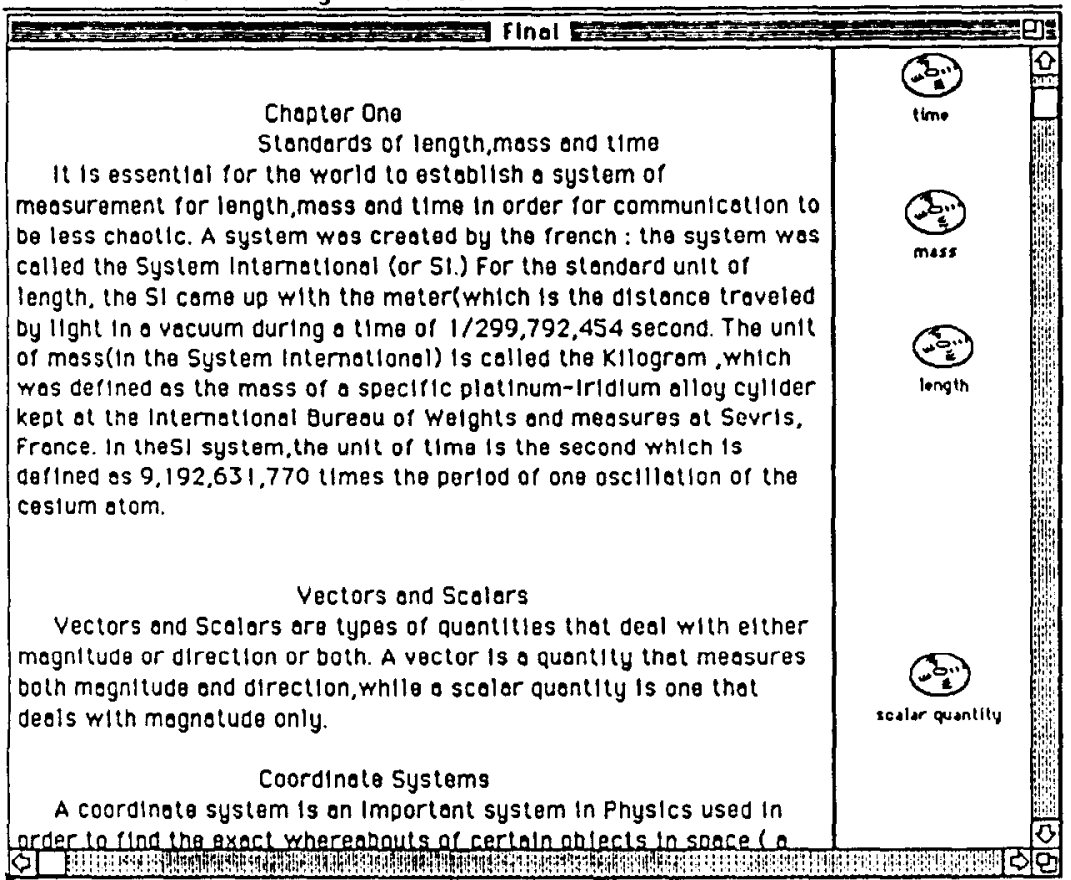

Fig. 3. Example of an integrated composition 
- Flle EdIt Font style Medialinks

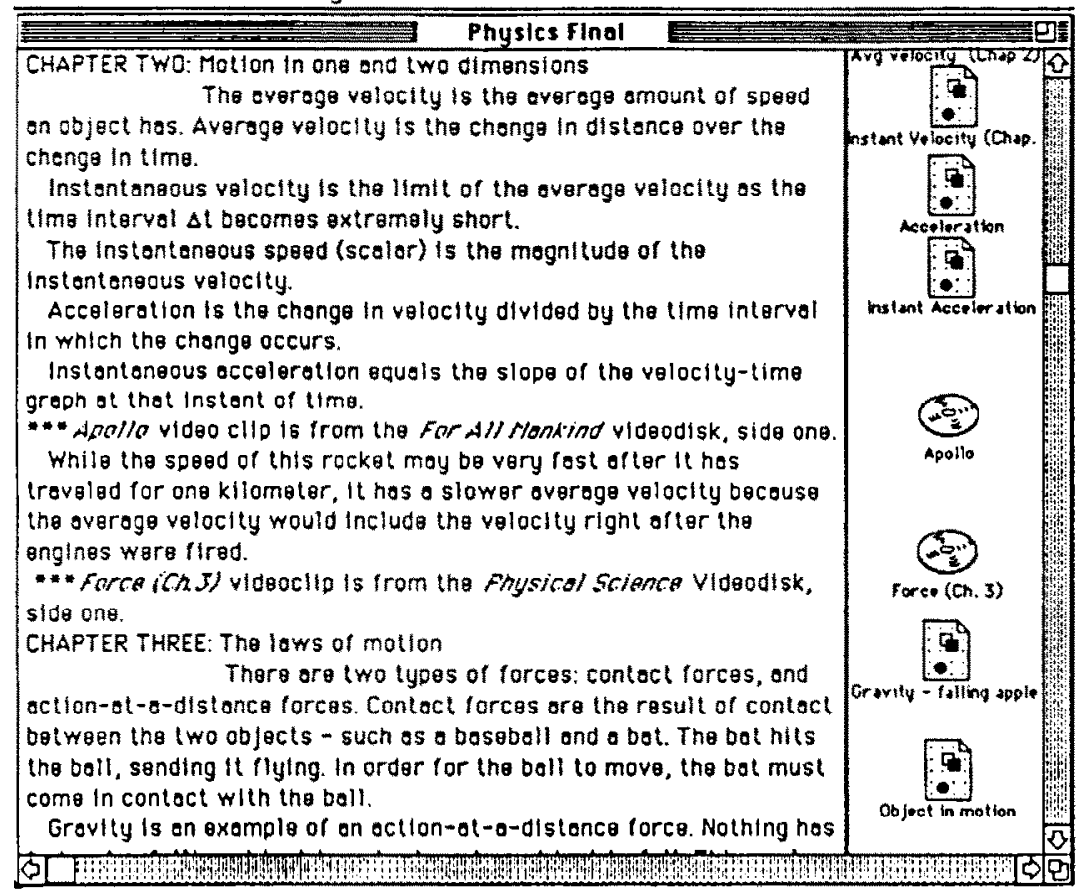

Pig. 4. Lixample of an integrated composition.

documents in the initial assignment one $\mathrm{AT}$ document and four $\mathrm{IC}$ documents in the final assignment.

From the analysis of the documents from these classes, a trend appears to emerge indicating that less experience with Mediatext results most often in AT style documents. Conversely, with greater experience, one might expeet to see more IC-style documents. However, two caveats need to be considered. The first is that some students did not finish later assignments, so our observation of trends should be considered tentative at this point. The second is that the assignments in this Multimedia course were intended to explicitly explore multimedia writing, as opposed to the other courses which were in traditional content areats. Thus, the focus of the class and requirements of the assignments may have influenced the increased number of IC documents. This suggests that more than experience is involved in determining the style in which a student will write.

In general, inexperienced users given a short period of time to complete their writing will persist with a style they know-lext-only essays. On the other hand, experience is not the only factor that will lead to multimedia compositions. Task factors, such as the purpose of the assignment. the structure of the assignment, availability of appropriate materials, or the amount of time allowed to complete the assignment, may also play a role, as may individual difrerences between students.

\section{Comparisons between students and their decuments}

The development of student cases focuses on three of the more experienced students. This allows us to examine similarities and differences between these students on the same assignments as well as between their own writings over a number of tasks and documents.

The students are referred to as Students A. B, and C. all of whom were members of the Multimedia: Art and Technology class. Additional selected documents were collected from each of the students' other experiences using MediaText. Student A was also in the Composition class, 
Table 4. Three individual students" work

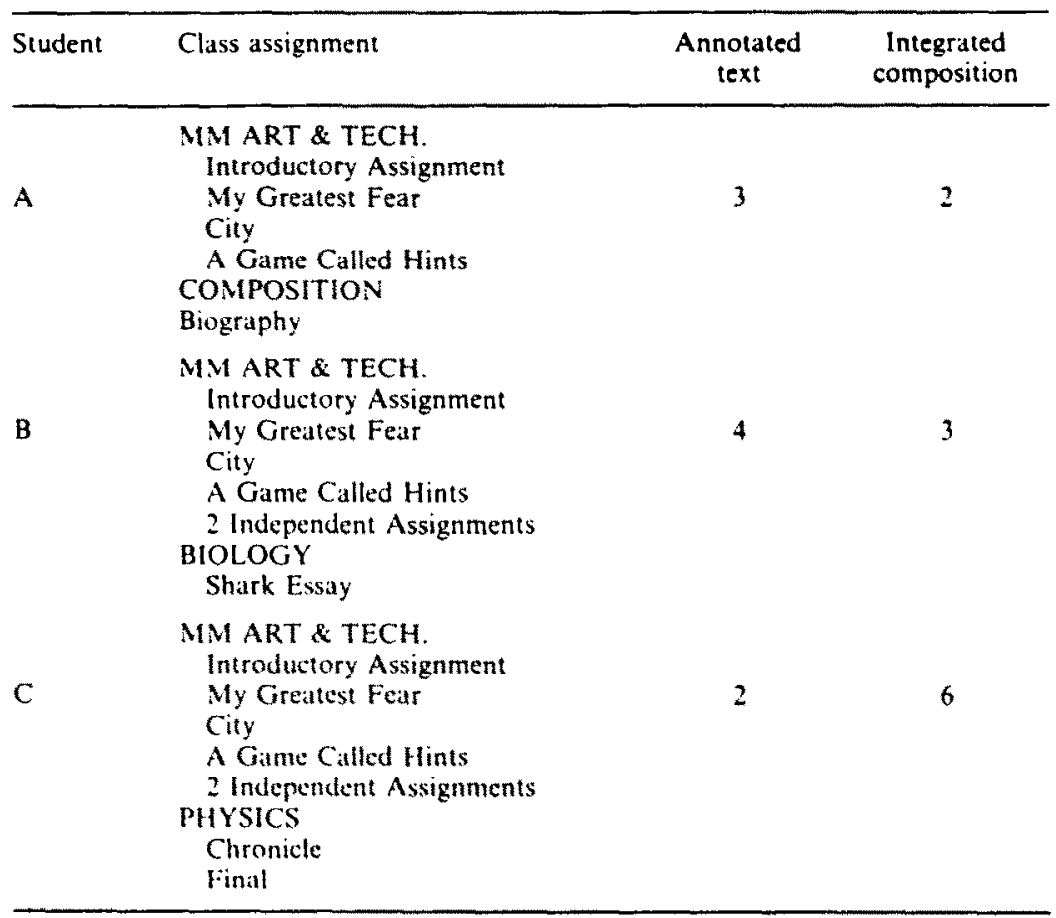

Student $B$ used MediaText in a Biology class, and Student $C$ was in the Physics class. Table 4 lists the classes and documents that were a part of our analysis, as well as, a breakdown of $\mathrm{AT}$ and IC documents.

\section{Student-by-student interpretation}

All of Student A's documents were structurally conventional. The text of his AT documents could easily stand apart from the links. In his IC documents, even though the structure of the sentences and paragraphs were conventional, the links played a central role in meaning construction. He used juxtaposition of links, directing the reader, in his text, to activate a particular link, and, to a lesser extent, discussed the content of links in his text.

Student B's documents were also conventional with the exception of one IC document (Fig. 5). In this document, the text relies heavily on the links to create the message and some links provide information not found in the text, but conceptually related to it. Student B used implicit integration techniques of juxtaposition and the explicit integration techniques of mentioning and directing.

Student $C$ displayed the greatest range in use of writing conventions. Some of his documents looked conventional and able to stand alone, while others had structured the text around extended discussion of links and their content. The most unconventional document was one that had no text whatsoever (Fig. 6)--it was comprised solely of links. Student $C$ used the integration techniques of justaposition most of the time, but was also more directing, in terms of activating links. His directing was more likely to engage in extended discussions of links than the directing used by the other students.

Generally, these three students demonstrated the general tendencies of all the students: a reliance on familiar sentence and paragraph structures in the creation of their documents and on the use of implicit integration of links and text through juxtaposition. This was true for both AT and IC documents. When they did use explicit integration techniques, it was primarily in the form of directions to click links or discussions of links. Where they did discuss link content in detail, their style tended towards IC rather than AT. 
* Flle Edit Font style Medlolinks

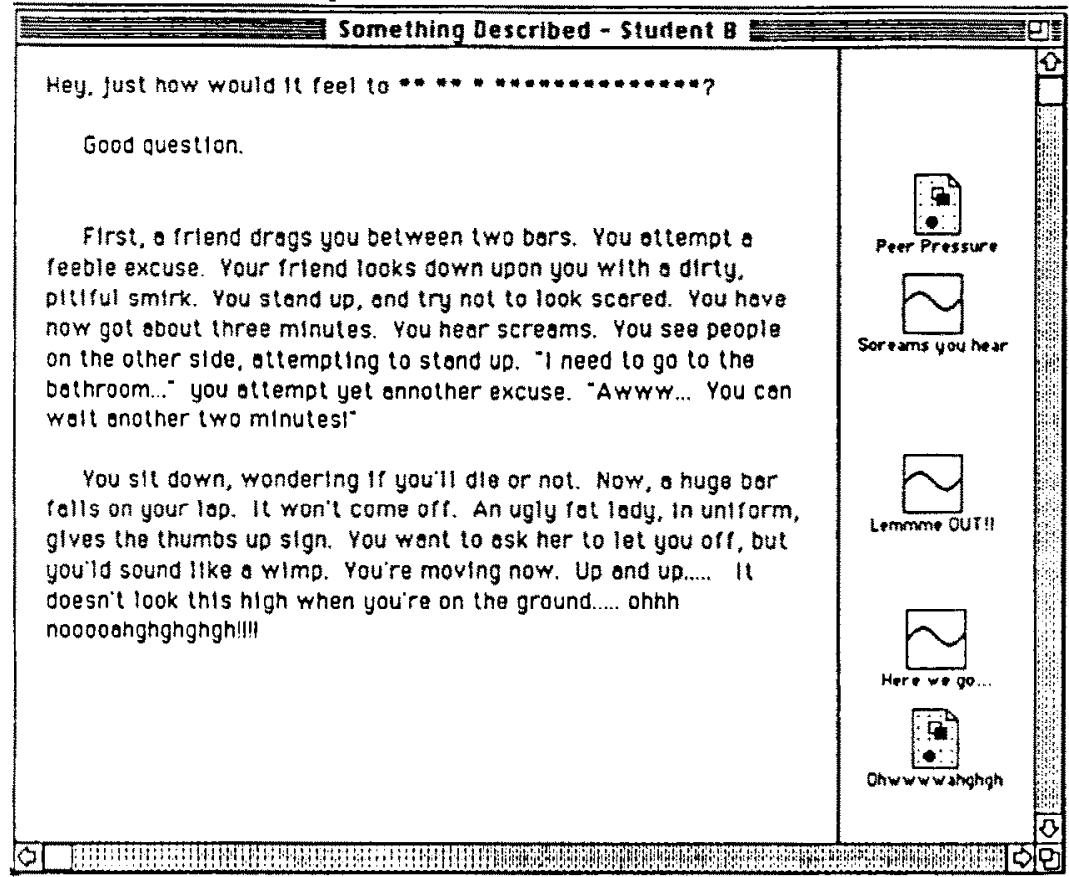

Fig. 5. Integrated composition document showing implicit and explicit integration.

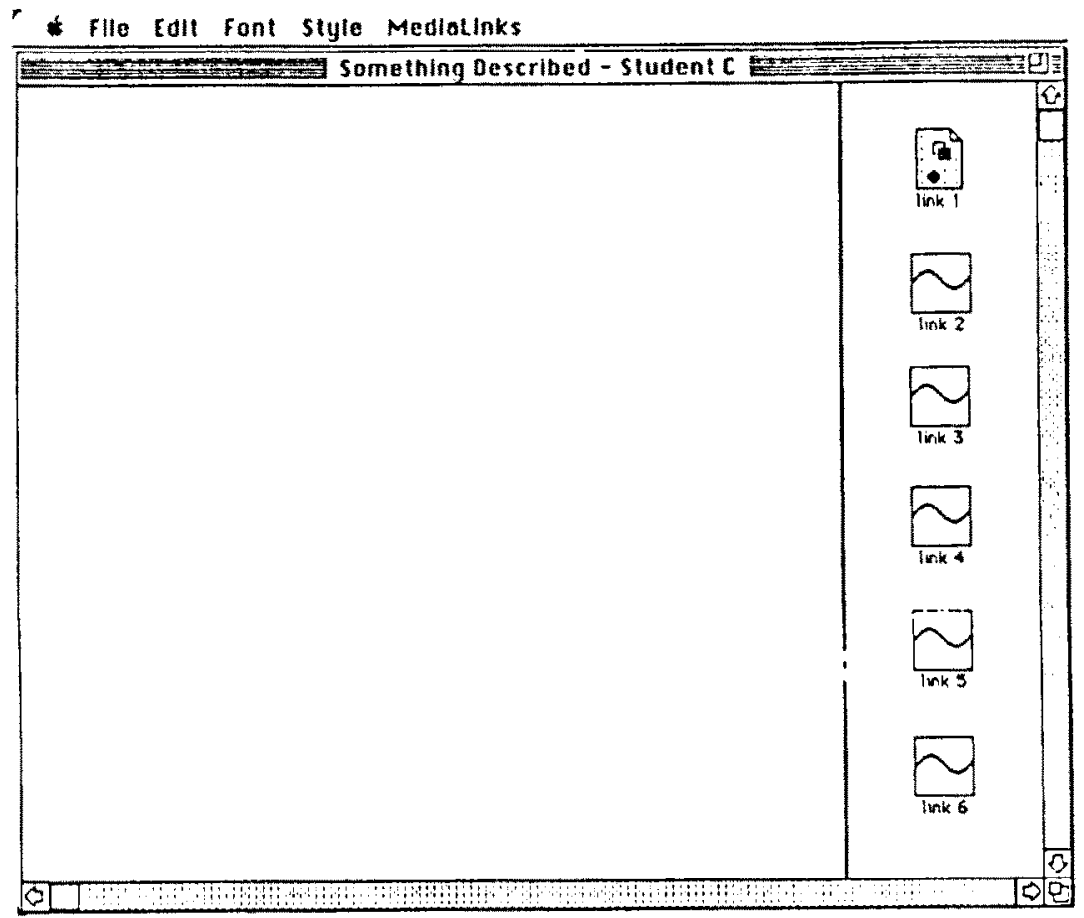

Fig. 6. Unconventional document showing only links. 


\section{Assignment-by-assignment interpretations}

All three introductory assignments produced were AT documents. Students $A$ and $C$ wrote IC documents for My Greatest Fear assignment. These documents were still fairly conventional in structure, although the content of the links was discussed in more depth. Students B and C wrote IC documents for the City assignment. Once again, their writing style was conventional. Student $\mathrm{B}$, like $\mathrm{A}$ and $\mathrm{C}$ in the previous assignment, discussed the links in detail. The beginning of Student C's document could be classified as AT, but in the course of writing, he discovered a source of information in other media. The second half of his document utilized this alternative medium and the document changed into an IC document where the non-text media began carrying the entire message. For the last assignment, A Game Called Hints (Fig. 5), all three produced IC documents. The meanings in all three of these documents were heavily dependent on their links. Although Student A's work remained conventional in structure, the links are integral to the document. The text and the links in Student B's document seem to be unrelated at first glance. Only by activating the links does the relationship between links and text, and the meaning of the piece, become apparent. Student C's A Game Called Hints is the document without text, conveying his message through the content of his animation and sound links.

Several observations can be made from the above interpretations. The first is the apparent similarity of the first documents. even though both Students $A$ and $C$ had used MediaText before. Student $C$ rather extensively. This reflects the students' reliance on familiar writing styles when faced with a novel situation. A second observation is, even for experienced users, multimedia composition does not necessarily imply unconventional structure. Often their IC, as well as AT, documents structurally looked like a normal essay. It appears that, as they gained experience, they tended to write more in the IC style. This may be somewhat misleading because the four assignments discussed above are not all of the documents these students produced; rather, they are four assignments that they all have in common. In conclusion, the factors, beyond experience, that seem to have some influence are: (1) the demands of the assignment, and (2) the availability of appropriate materials in various media formats related to the topies of documents.

\section{DISCUSSION}

This work on student use of multimedial composition tools is still in its exploratory stage. The data came mostly from a year which involved the continuing development of the software tool, based mostly on suggestions from the students using it. As such, the implementation of the software in these classes took an unstructured form. Rather than dictating a proper use of the software, we let the students make what use of it that they would, constructing their own styles of multimedia composition.

Within this exploratory frame, we have found there are several promising threads that merit further attention. In particular, there is the issue of the difference between writing as Annotated Text and as Integrated Composition. As mentioned earlier, there was very little structure in terms of how students using MediaText should compose their documents. The students were, for the most part, given free rein to use whatever media they preferred and to integrate these media to the extent they saw fit. That these two styles should arise out of the student's work suggests that different processes of knowledge construction may be taking place.

In particular, several aspects of integrated composition appear to relate to issues involving the encoding of information. As cited in the rationale for the student composition of multimedia documents, by using multiple representations in dilferent media formats, students constructed a richer understanding of the concepts underlying these representations. When the student discusses the relationship between the content of a link and the content of a section of text, it may also aid the student in constructing meaningful representations. This can be facilitated by providing a variety of resource materials to help students find concrete examples of abstract principles that they find meaningful. As an example, one student in physics used a scene from 2001: A Space Odyssey to demonstrate Newton's First Law of Motion.

As students integrate Links and their text, as in the assignment A Game Called Hints, the documents show that they began to think about the interconnections between the ideas expressed CAE 2J: A-E 
in the different media. Students can also find concrete examples of abstract ideas in unusual places. In another example from physics, a student used scenes from a videodisk collection of art to illustrate items approximately the size of certain units of measure, e.g. a pearl having a mass of close to $1 \mathrm{~g}$. Rather than looking for examples in science fiction movies. she and another student drew examples from The Wizard of $O$ z and Who Framed Roger Rabbit? to illustrate mechanical principles and laws. In explaining the difference between initial, final, average, and instantaneous velocity, she instructed the reader in how to manipulate the videodisk player and what to observe to see each of these concepts illustrated.

With respect to motivational issues, MediaText allows for active learning in a multimedia environment. One student described his experiences in an interview,

"I'm actually learning physics for the first time this semester ... because I can see what's happening with the concepts. They're not just in a book, they're really happening... I'm also teaching myself ... which. I guess, is the best way to learn something."

Another student described how the ability to use different media influenced the specific choices he made when trying to decide on a topic to write about, emphasizing visual or sound media for subjects that fit best with either type. Finally, all the students and the teachers we worked with were excited about the opportunities for alternative assessment that multimedia creates. For some of the students who chose to do the final paper in Physics, the notion of an in-class exam heavy in calculation problems was not only daunting. but it did not seem to address the way they understood the matterial best. Through multimedia compositions, they were able to deal conceptually with the content using the ditlerent representation. thus they were not stuck in Physics traditional exclusive mathematical lramework.

Concerns arose from the nature of multimedia itself. Though, for this initial project, we did not impose any overall structure upon the students' activities or assigmments with MediaText, structured activities or specific models would help focus the students on particular styles of multimedia composing. for example, in a majority of the cilses, students did not engage in extended deliberations about the content or selection of their links. Instead, the lack of structure and/or models led to a number of students just pulting media together without much thought. If model composition or structured activities were given students, they would have probably spent more time in deliberation. Another area in which reflection and deliberation of media choices could be optimized is in the availability of a wide variety of easily identifiable media available to the students. If stadents can be made aware of which media are best suited for the topic of their assignment, much time might be saved in searching these materials for possible links and more time would be spent on composing documents.

This study of student composition of multimedia documents takes the first steps towards applying the approach of a constructionist paradigm to look at the effects with the technology. Students can take the next step into new styles of writing if they are given the opportunity, the time, and experience with the technology.

Acknowlatgemonts - The HiCE group is tirected by Professor Elliot Soloway of the Department of Electrical Engineering and Computer Science. School of Engineering. at the University of Michigan. MediaText was conceived of by Mark Guzdial and developed primarily by Jim Mert, both students at the University of Michigan.

\section{REFERENCES}

1. Clark R. E., Reconsidering the research on laarning from media. Rev. Ealuc. Res. 53,445460 (1989).

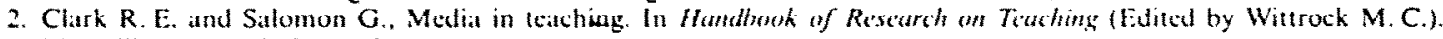
Macmillan, New York $(1985)$

3. Pintrich P. R., Cross D. R., Kozma R. B. and Mckeachie W. J., Instructional psychology. A. Re't: Psyc/w/. 37,611-615 $(1990)$.

4. Kouma R. B., Learning with media. Re's: Fstus: Re's. 61, 179-211 (1991).

5. Papert S. Introduction by Seymour Papert. In Comseructionist Learning (Edited by Harel I.). MITT Media Laboratory. Boston. Mass. (1990). 
6. Salomon G., Perkins D. N. and Globerson T., Partners in cognition; extending human intelligence with intelligent technologies. Echuc. Res. 20, No. 3. 2-9 (1991).

7. Neuwirth $C$. and Kaufer D. The role of external representations in the writing process: implications for the design of hypertext-based writing tools. In Hypertext ' 89 Proceedings. Association tor Computing Machinery. New York (1989).

8. Greeno J. Situations, mental models, and generative knowledge. In Complex Information Processing (Edited by Khahr D. and Kotovsky K.). Erlbaum, Hillsdale, N.). (1989).

9. Bransford I. D. Sherwood R. D. Hasselbring T. S., Kinzer C. K. and Williams S. M., Anchored instruction: why we need it and how technology can help. In Cognition. Education, and Multimedia (Edited by Nix D. and Spiro R.). Erlbaum. Hillsdale. N.J. (1990)

10. Scarmadalia M. Bereiter C., ICLean R.S., Swallow J. and Woodruff E.. Computer-supported intentional learning environments. J. Educ. Comput. Res. 5, $51-68$ (1989).

11. Lepper M., Microcomputers in education: motivational and social issues. Am. Pswchol, 40, 1-18 (1985).

12. Beekman G. Recreating Native American legends with HyperCard. Comput. Teucher 31 (1992).

13. Scheidler K.. Students cross-discipline boundaries with hypermedia. Comput. Teacher 16-20 (1993).

14. Goodman D.. The Complete HyperCard Handbook. Bantam, New York (1987).

15. Nicol A. Children's hypermedia compositions. I. Comput. Childhood Edur. 1, No. 2, 3-17 (1989/1990).

16. Turner S. V. and Dipinto V. M.. Students as hypermedia authors: themes emerging from qualitative study. J. Res. Compus. Educ. 25, 187-199 (1992).

17. Farrow M. Knowledge-engineering using HyperCard: a leaming strategy for tertiary education. $J$. Comput. hused Instrue. 20,9-14 (1993).

18. Reed M.W. and Rosenbluth. G.S. The effect of HyperCard programming on knowledge construction and inierrelatedness of humanities-based information. ERIC document Reproduction Service No. ED 355908 (1992).

19. Pea R.. Multimedia works: student learning through multimedia tools. IEE Conput. Graphics Applic. 11, No, 4, 58 66 (1991).

20. Rielly B., The Negotiations of Group Authorship Among Second Graders using Multimedia Composing Softwarc. Apple Computer Ine., Cupertino, Calif. (1992).

21. Dede C. The future of multimedia: bridging to virtual worlds. Educ. Technol. 32, No. 5, 54-60 (1992).

22. Strauss A. and Corbin I., Basic Qualitative Research: Growhded Theory. Pracedurey and Techniques. Sage, Newbury Park, Calif, (1990).

23. Lincoln Y. and Guba E. Naturalistic Inquiry. Sage. Beverly Hills, Calif, (1985). 